\title{
THE $Q$-TOPOLOGY, WHYBURN TYPE FILTERS AND THE CLUSTER SET MAP
}

\author{
ROBERT A. HERRMANN
}

\begin{abstract}
We use nonstandard topology and the $Q$-topology to characterize normal, almost-normal, regular, almost-regular, semiregular spaces. The cluster [resp. $\theta$-cluster] set relation is used to characterize regular, almostregular [resp. strongly-regular] spaces. The Whyburn [resp. Dickman] filter bases are characterized and it is shown that the cluster [resp. $\theta$-cluster] set relation restricted to the domain of the Whyburn [resp. Dickman] filter bases is an essentially continuous [resp. strongly $\theta$-continuous] map iff the space is Hausdorff [resp. Urysohn].
\end{abstract}

1. Introduction. This paper has three major purposes. First, we investigate the $Q$-topology on an enlargement ${ }^{*} X$ of a topological space $X$ as introduced by Robinson [9] and show, among other results, that the $Q$-closure of a point or set monad is the $\theta$-monad [6]. Moreover, using the $Q$-topology and the point or set monad, we characterize regular, semiregular, almost-regular [10], normal and almost-normal [11] spaces by means of a collection of highly analogous statements.

Fuller [4] defines a topology on the set of all clustering filters on $X$ and using the lower semifinite topology shows that the cluster set map is continuous iff $X$ is locally compact. Employing a different topology on the set of all converging filters, Wyler [13] shows that the convergence of a filter on a Hausdorff space $X$ is a continuous map iff $X$ is regular. We use that standard part [resp. $\theta$-standard part] relation, which can be considered the cluster [resp. $\theta$-cluster] set map, and show that, from the nonstandard viewpoint, regular [resp. almost-regular, strongly-regular] spaces are characterizable by similar statements involving the inverse of this relation. Further, by considering the near-standard [resp. $\theta$-near-standard] points and employing the induced $Q$ topology, we show that the cluster [resp. $\theta$-cluster] set relation is a continuous map iff $X$ is Hausdorff [resp. Urysohn].

In [12], Whyburn introduces the concept of a filter base being directed toward $A \subset X$ and uses this concept to characterize perfect (not necessarily continuous) maps. Dickman [2], [3] modifies Whyburn's definition and introduces the concept of a filter base almost-converging to $A \subset X$. Among our final results, we show that a filter base is directed toward [resp. almostconverges to] $A \subset X$ iff its nucleus satisfies a nonstandard condition analo-

Presented to the Society, January 24, 1976; received April 23, 1975.

AMS (MOS) subject classifications (1970). Primary 54J05; 54D99; Secondary 02H25.

Key words and phrases. $Q$-topology, Whyburn filters, Dickman filters, regular, almost-regular, strongly-regular, semiregular, normal, almost-normal, cluster set map, $\theta$-cluster set map, directed toward, almost-convergent to. 
gous to the criterion for compactness [resp. quasi- $H$-closedness].

Throughout this paper, we let $\mathscr{R}=(\mathscr{Q}, \in, \mathrm{pr}$, ap $)$ be the standard settheoretic structure constructed by Machover and Hirschfeld [8] and, as usual, assume that all standard objects are elements of 2 . Even though some of the results only require ${ }^{*} \Re=\left({ }^{*}{ }_{\mathscr{Q}},{ }^{*} \in,{ }^{*} \mathrm{pr},{ }^{*}\right.$ ap $)$ to be an enlargement, it is convenient to assume that the extension ${ }^{*} \Re$ is $\kappa$-saturated, where $\kappa$ is any cardinal larger than the cardinality of $\Re$. In the usual manner [7], [8], [9] we let $\mathcal{E}$ be a first order language with equality and the usual assortment of abbreviations which formally describes $\Re$. Also we do not distinguish between the formal constant, relation and operator symbols in $\mathcal{L}$ and the corresponding objects in $\Re$. We assume that the reader is familiar with the concepts and methods associated with nonstandard topology [7], [8], [9]. We use much of the notation found in [8].

2. The $Q$-topology. For a topological space $(X, \tau)$, the $Q$-topology on ${ }^{*} X$, denoted by $\mathcal{T}$, is the topology generated by $\left\{{ }^{*} A \mid A \in{ }^{*} \tau\right\}$ as a base. Recall that if $A \in{ }^{*}$ Q , then ${ }^{*} A=\left\{p \| p \in{ }^{*}\right.$ U] $\left.\wedge\left[p \in \in^{*} A\right]\right\}$. If $A \in \in^{*} \tau$, then ${ }^{*} A$ is said to be ${ }^{*}$-open. If $B \in \mathcal{T}$, then $B$ is said to be $Q$-open, etc. We let $\mu(p)$ and $\mu(A)$ be the point and set monad [9] and define

$$
\begin{aligned}
& \mu_{\alpha}(p)=\bigcap\left\{{ }^{*}\left(\text { int }_{X} \mathrm{cl}_{X} G\right) \mid p \in G \in \tau\right\}, \\
& \mu_{\alpha}(A)=\cap\left\{{ }^{*}\left(\text { int }_{X} \mathrm{cl}_{X} G\right) \mid A \subset G \in \tau\right\}, \\
& \mu_{\theta}(p)=\bigcap\left\{{ }^{*}\left(\mathrm{cl}_{X} G\right) \mid p \in G \in \tau\right\}, \\
& \mu_{\theta}(A)=\bigcap\left\{{ }^{*}\left(\mathrm{cl}_{X} G\right) \mid A \subset G \in \tau\right\}
\end{aligned}
$$

to be the $\alpha$ and $\theta$ point and set monads respectively.

For many properties of the $Q$-topology not mentioned in this paper, we refer the reader to [1], [9]. In particular, Button [1] has shown that the $Q$-topology preserves much of the structure of $\tau$ and, indeed, $\left({ }^{*} X, \mathcal{T}\right)$ is discrete iff $(X, \tau)$ is discrete.

THEOREM 2.1. If nonempty $\mathcal{G} \subset \tau$, then $\operatorname{Nuc} \mathcal{G}$ is $Q$-open.

Proof. If $\mathcal{G}$ does not have the finite intersection property, then $\operatorname{Nuc} \mathcal{G}=\varnothing$. Assume that $\mathcal{G}$ has the finite intersection property and let $\mathscr{F}$ be the open filter generated by $\mathcal{G}$. Luxemburg's Theorem 2.1.6 [7] holds for any filter on any meet-semilattice of sets [5]. Hence Nuc $\mathcal{G}=\cup\left\{{ }^{*} E \mid\left[E \in{ }^{*} \mathscr{F}\right] \wedge\left[{ }^{*} E\right.\right.$ $\subset$ Nuc 9 ]\}.

Corollary 2.1.1. For each $p \in X$ and $A \subset X$, the monads $\mu(p), \mu(A), \mu_{\alpha}(p)$, $\mu_{\alpha}(A)$ are $Q$-open.

REMARK. In [1], Button obtains 2.1 by using a considerably more elaborate technique.

Clearly, if $\mathcal{G}$ is an open filter on $X$, then every infinitesimal *element in $\mathcal{G}$ is * open. Indeed, we have a converse to this assertion.

THEOREM 2.2. Let $\mathcal{F}$ be a filter base on $X$. If each infinitesimal *element in $\mathcal{F}$ is *-open, then Nuc $\mathcal{F}=$ Nuc $\mathcal{G}$, where $\mathcal{G}=\{G \mid[G \in \tau] \wedge[G \in \mathscr{F}]\}$. 
Proof. Since $\mathscr{F}$ is a filter base, then there exists an infinitesimal *element in $\mathscr{F}$. Thus, it follows by transfer that $\mathcal{G}=\{G \mid[G \in \tau] \wedge[G \in \mathscr{F}]\} \neq \varnothing$. Clearly, Nuc $\mathcal{F} \subset$ Nuc $\mathcal{G}$. Now let $F \in \mathscr{F}$ and $\mathcal{Q}=\left\{E \| E \in{ }^{*} \tau\right] \wedge\left[E \in{ }^{*}{ }_{\mathscr{F}}\right] \wedge\left[{ }^{*} E\right.$ $\left.\left.\subset^{*} F\right]\right\}$. Using saturation and Luxemburg's Theorem 2.7.3(c) [7], which also holds for filter bases, we have that there exists an open $G \in \mathcal{F}$ such that $G \subset F$. Consequently, Nuc $\mathcal{G} \subset$ Nuc $\mathscr{F}$ and the result follows.

Clearly, for $A \subset X,{ }^{*}\left(\operatorname{cl}_{X} A\right)$ is *-closed. Hence $\mu_{\theta}(p)$ and $\mu_{\theta}(A)$ are $Q$ closed. Of course, ns $\left({ }^{*} X\right)=\cup\{\mu(p) \mid p \in X\}$ is $Q$-open.

Theorem 2.3. For each $p \in X[$ resp. $A \subset X]$, the monad $\mu_{\theta}(p)=\operatorname{cl}_{*_{X}}(\mu(p))$ $\left[\right.$ resp. $\left.\mu_{\theta}(A)=\operatorname{cl}_{*_{X}}(\mu(A))\right]$.

Proof. We only show the first assertion, the second being similar. Let $p \in X$. Since $\mu(p) \subset \mu_{\theta}(p)$, then $\operatorname{cl}_{*_{X}}(\mu(p)) \subset \mu_{\theta}(p)$. Assume that there exists $q \in \mu_{\theta}(p)$ and $q \notin \operatorname{cl}^{*} X(\mu(p))$. Now there exists $E \in{ }^{*} \tau$ such that $q \in{ }^{*} E$ and ${ }^{*} E \cap \mu(p)=\varnothing$. Saturation implies that there exists $G \in \tau$ such that $p \in G$ and ${ }^{*} E \cap{ }^{*} G=\varnothing$. Hence ${ }^{*} E \cap{ }^{*}\left(\mathrm{cl}_{X} G\right)=\varnothing$ by transfer. However, $q \in \mu_{\theta}(p)$ implies ${ }^{*} E \cap{ }^{*}\left(\mathrm{cl}_{X} G\right) \neq \varnothing$ and the result follows.

Since $X$ is regular [resp. almost-regular [10]] iff $\mu(p)=\mu_{\theta}(p)$ [resp. $\mu_{\alpha}(p)$ $\left.=\mu_{\theta}(p)\right]$ for each $p \in X[6]$, then it follows that a space $X$ is regular [resp. almost-regular] iff $\mu(p)$ [resp. $\mu_{\alpha}(p)$ ] is $Q$-closed for each $p \in X$. Also, it is easy to show that a space $X$ is normal [resp. almost-normal [11]] iff $\mu(A)=\mu_{\theta}(A)$ [resp. $\left.\mu_{\alpha}(A)=\mu_{\theta}(A)\right]$ for each closed $A \subset X$. Hence a space $X$ is normal [resp. almost-normal] iff $\mu(A)$ [resp. $\mu_{\alpha}(A)$ ] is $Q$-closed for each closed $A \subset X$.

REMARK. Button [1], using a different technique, also gives the $Q$-open and $Q$-closed characterizations for regular and normal spaces.

In [6], we give some nonstandard characterizations for semiregular spaces. Using the $Q$-topology, we obtain another characterization. Let $\tau_{s}$ be the topology generated by the set of all regular-open subsets in $X$ and $\mathscr{T}_{s}$ its associated $Q$-topology.

Theorem 2.4. A space $(X, \tau)$ is semiregular iff $\mu(p) \in \mathcal{T}_{s}$ for each $p \in X$.

Proof. For the necessity, let $(X, \tau)$ be semiregular. Then $\mathcal{T}_{s}=\mathcal{T}_{\text {. Thus }}$ applying 2.1.1, we have that $\mu(p) \in \mathcal{T}_{s}$ for each $p \in X$.

For the sufficiency, let $\mu(p) \in \mathcal{T}_{s}$. Since ${ }^{*} \tau_{s}$ is a base for $\mathcal{T}_{s}$ and $p \in \mu(p)$, then it follows that there exists $E \in{ }^{*} \tau_{s}$ such that $p \in{ }^{*} E \subset \mu(p) \subset \mu_{\alpha}(p)$. Let $G$ be any open set such that $p \in G$. Then the sentence in $\rho$,

$$
\exists x\left[\left[x \in \tau_{s}\right] \wedge[p \in X] \wedge[x \subset G]\right],
$$

holds in $\mathfrak{N}$ by transfer. Consequently, since we are dealing with filter bases, we have that $\mu_{\alpha}(p) \subset \mu(p)$. Thus $\mu(p)=\mu_{\alpha}(p)$. This implies that $(X, \tau)$ is semiregular [6].

3. The cluster set map. As is well known if $\mathscr{F}$ is a filter base on $X$, then St [NucF $\mathcal{F}$ is the cluster set for $\mathscr{F}$, where for $W \subset^{*} X$, St $[W]=\{p \|[p \in X]$ $\wedge[\mu(p) \cap W \neq \varnothing]\}$. Recall that a set $W \subset^{*} X$ is nuclear if there exists $\mathscr{F} \subset \mathcal{P}(X)$ such that $W=$ Nuc $\mathscr{F}$. Hence "St" restricted to ns $\left({ }^{*} X\right)$ is essentially the cluster set map for filter bases on $X$. Of course, in this case "St" may be considered a map from ns $\left({ }^{*} X\right)$ into $X$ iff $X$ is Hausdorff. A space $(X, \tau)$ is 
called strongly-regular if for closed $F \subset X$ and $p \in X-F$ there exist $G, H$ $\in \tau$ such that $p \in G, F \subset H$ and $\operatorname{cl}_{X} G \cap \mathrm{cl}_{X} H=\varnothing$. Observe that completely regular implies strongly-regular implies regular.

Definition 3.1. For each $W \subset^{*} X$, let $\mathrm{St}_{\theta}[W]=\left\{p \mid[p \in X] \wedge\left[\mu_{\theta}(p)\right.\right.$ $\cap W \neq \varnothing]\}$ and $\mathrm{ns}_{\theta}\left({ }^{*} X\right)=\bigcup\left\{\mu_{\theta}(p) \mid p \in X\right\}$. Notice that if $\mathcal{F}$ is a filter base, then $\mathrm{St}_{\theta}$ [Nuc $\mathcal{F}$ ] is the set of all $\theta$-cluster points [3] for $\mathscr{F}^{\text {. Also, " }} \mathrm{St}_{\theta}$ " is a map from $\mathrm{ns}_{\theta}\left({ }^{*} X\right)$ into $X$ iff $X$ is Urysohn [6] (i.e. for distinct $p, q \in X$ there exist neighborhoods $N_{p}, N_{q}$ such that $\left.\mathrm{cl}_{X} N_{p} \cap \mathrm{cl}_{X} N_{q}=\varnothing\right)$.

TheOREM 3.1. Let $(X, \tau)$ be Hausdorff and St : ns $\left({ }^{*} X\right) \rightarrow X$. Then:

(i) $X$ is regular iff $\mathrm{St}^{-1}[F]=\mu(F) \cap \mathrm{ns}\left({ }^{*} X\right)$ for each closed $F \subset X$.

(ii) $X$ is almost-regular iff $\mathrm{St}^{-1}[F]=\mu_{\alpha}(F) \cap \mathrm{ns}\left({ }^{*} X\right)$ for each regularclosed $F \subset X$.

Proof. (i) For the necessity, let closed $F \subset X$ and $q \in \mathrm{St}^{-1}[F]$. Then St $(q)=p$ implies that $q \in \mu(p)$ and $\mu(p) \cap{ }^{*} F \neq \varnothing$. Hence $p \in F$. Thus $\mu(p) \subset{ }^{*} G$ for each open $G \supset F$. Consequently, $q \in \mu(F) \cap$ ns $\left({ }^{*} X\right)$ implies that $\mathrm{St}^{-1}[F] \subset \mu(F) \cap \mathrm{ns}\left({ }^{*} X\right)$. Now assume that $X$ is regular and $q$ $\in \mu(F) \cap \mathrm{ns}\left({ }^{*} X\right)$. Then $q \in \mu(p)$ for some $p \in X$. Assume that $p \notin F$. Then there exist disjoint $G, H \in \tau$ such that $p \in G$ and $F \subset H$. Thus $\mu(p) \cap{ }^{*} H=\varnothing$. However, this implies the contradiction that $q \notin \mu(F)$. Consequently, St $[\mu(p)]=p \in F$ and the necessity follows.

For the sufficiency, let closed $F \subset X$ and $p \notin F$. Then $\mathrm{St}^{-1}(p)=\mu(p)$ $\subset$ ns $\left({ }^{*} X\right)$ and $\mathrm{St}^{-1}[F]=\mu(F) \cap$ ns $\left({ }^{*} X\right)$. Observe that $\mu(p) \cap{ }^{*} F=\varnothing$. Hence

$$
\begin{aligned}
\varnothing & =\mathrm{St}^{-1}[F \cap\{p\}]=\mathrm{St}^{-1}[F] \cap \mathrm{St}^{-1}(p) \\
& =\mu(F) \cap \mathrm{ns}\left({ }^{*} X\right) \cap \mu(p)=\mu(F) \cap \mu(p) .
\end{aligned}
$$

Thus there exist disjoint $G, H \in \tau$ such that $p \in G$ and $F \subset H$.

(ii) Observe that if $F \subset X$ is regular-closed in $X$, then

$$
\mathrm{St}^{-1}[F] \subset \mu(F) \cap \mathrm{ns}\left({ }^{*} X\right) \subset \mu_{\alpha}(F) \cap \mathrm{ns}\left({ }^{*} X\right) .
$$

The result follows in the same manner as in (i) since the operator "int ${ }_{X} \mathrm{cl}_{X}$ " preserves disjointness for open sets.

Clearly, a strongly-regular $T_{1}$ space is Urysohn. Of course, since a stronglyregular space is regular, then in a strongly-regular space $X, F \subset X$ is closed iff $\mathrm{St}_{\theta}\left[{ }^{*} F\right]=F$. The following result is obtained in the same manner as is Theorem 3.1.

THeOREM 3.2. Let $X$ be Urysohn. Then $X$ is strongly-regular iff $\mathrm{St}_{\theta}^{-1}[F]$ $=\mu_{\theta}(F) \cap \operatorname{ns}_{\theta}\left({ }^{*} X\right)$ for each closed $F \subset X$.

4. Whyburn and Dickman filter bases. In [12], Whyburn says that a filter base $\mathcal{F}$ on $X$ is directed toward $A \subset X$ if every filter base $\mathcal{G}$ stronger than $\mathscr{F}$ has a cluster point in $A$. Dickman [3] modifies Whyburn's definition and says that a filter base $\mathscr{F}$ on $X$ is almost-convergent to $A \subset X$ if every filter base $\mathcal{G}$ stronger than $\mathscr{F}$ has an almost-cluster point in $A$ (i.e. $\mathrm{St}_{\theta}$ [Nuc $\left.\mathcal{G}\right] \cap A \neq \varnothing$ ). We call a filter base $\mathscr{F}$ a Whyburn [resp. Dickman] filter base if $\mathscr{F}$ is directed toward [resp. almost-convergent to] some $A \subset X$. 
Definition 4.1. A set $W \subset^{*} X$ is $A$-compact [resp. $\theta A$-compact] for $A \subset X$ if $W \subset \cup\{\mu(p) \mid p \in A\}$ [resp. $\left.\left\{\mu_{\theta}(p) \mid p \in A\right\}\right]$.

TheOREM 4.1. Let $\mathscr{F}$ be a filter base on $X$. Then the following statements are equivalent.

(i) For each open cover $\mathcal{C}$ of $A$, we have that $\operatorname{Nuc} \mathcal{F} \subset \cup\left\{{ }^{*} G \mid G \in \mathcal{C}\right\}[$ resp. $\left.\left\{*\left(\mathrm{cl}_{X} G\right) \mid G \in \circlearrowright\right\}\right]$.

(ii) Nuc F is A-compact [resp. $\theta A$-compact].

(iii) For each open cover $C$ of $A$ there exists a finite subcover $\mathscr{D}$, such that Nuc $\mathcal{F} \subset \cup\left\{{ }^{*} D \mid D \in \mathscr{D}\right\}\left[\right.$ resp. $\left.\left\{{ }^{*}\left(\operatorname{cl}_{X} D\right) \mid D \in \mathscr{D}\right\}\right]$.

(iv) For each open cover $C$ of $A$ there exists a finite subcover $\mathscr{D}$ and an $F \in \mathscr{F}$, such that $F \subset \cup\{D \mid D \in \mathscr{D}\}\left[\right.$ resp. $\left.\left\{\operatorname{cl}_{X} D \mid D \in \mathscr{D}\right\}\right]$.

Proof. We only prove the first conclusions since the second follow in a similar manner.

(i) $\rightarrow$ (ii). Assume that $q \in \operatorname{Nuc}^{\mathcal{F}}$ and $q \notin \cup\left\{\mu_{\theta}(p) \mid p \in A\right\}$. Then for each $p \in A$ there exists some open neighborhood $G$ such that $q \notin{ }^{*} G$. Thus $\mathcal{e}=\left\{G \mid[G \in \tau] \wedge\left[q \notin^{*} G\right]\right\}$ is an open cover of $A$ such that Nuc $\mathscr{F}$ $\varpi \cup\left\{{ }^{*} G \mid G \in \mathcal{C}\right\}$.

(ii) $\rightarrow$ (iii). Assume that there exists some open cover $\bigodot$ of $A$ such that for no finite $\mathscr{D} \subset \mathcal{C}$ do we have that Nuc $\mathscr{F} \subset \cup\left\{{ }^{*} D \mid D \in \mathscr{D}\right\}$. Now there exists internal $E^{*} \in \mathscr{F}$ such that ${ }^{*} E \subset$ Nuc $\mathscr{F}$ and

$$
{ }^{*} E-\cup\left\{{ }^{*} D \mid D \in \mathscr{D}\right\} \neq \varnothing
$$

for any finite $\mathscr{D} \subset \mathcal{C}$. For if we assume that

$$
{ }^{*} E-\cup\left\{{ }^{*} D \mid D \in \mathscr{D}\right\}=\varnothing
$$

for some nonempty finite $\mathscr{D} \subset \mathcal{C}$, where $|\mathscr{D}|=n$, then the sentence in $C$,

$$
\exists x\left[[x \in \mathscr{F}] \wedge\left[x \subset D_{1} \cup \cdots \cup D_{n}\right]\right],
$$

holds in ${ }^{*} \mathfrak{T}$. Thus by transfer there would exist $F \in \mathscr{F}$ such that ${ }^{*} F$ $\subset^{*}\left(D_{1} \cup \cdots \cup D_{n}\right)={ }^{*} D_{1} \cup \cdots \cup{ }^{*} D_{n}$. This would imply the contradiction that $\mathrm{Nuc}^{*} \subset^{*} F \subset{ }^{*} D_{1} \cup \cdots \cup{ }^{*} D_{n}$. Consequently, using saturation, ${ }^{*} E-\cup\left\{{ }^{*} C \mid C \in \mathcal{C}\right\} \neq \varnothing$ implies that there exists $q \in{ }^{*} E \subset$ Nuc $\mathscr{F}$ such that $a \notin^{*} C$ for any $C \in \mathcal{C}$ and the result follows.

(iii) $\rightarrow$ (iv). Simply let $E$ be the infinitesimal *element which is contained in $*_{\mathscr{F}}$. Then the sentence in $\varrho$,

$$
\exists x[[x \in \mathscr{F}] \wedge[x \subset \cup\{D \mid D \in \mathscr{D}\}]],
$$

holds in $* \Re$; hence in $\Re$ by transfer.

(iv) $\rightarrow$ (i) is obvious.

COROllary 4.1.1. A filter base $\mathscr{F}$ on $X$ is Whyburn [resp. Dickman] iff Nuc $\mathscr{F} \subset \mathrm{ns}\left({ }^{*} X\right)\left[\right.$ resp. Nuc $\left.\mathcal{F} \subset \mathrm{ns}_{\theta}\left({ }^{*} X\right)\right]$.

Corollary 4.1.2. A filter base $\mathcal{F}$ on $X$ is directed toward [resp. almostconverges to] $A \subset X$ iff Nuc $\mathscr{F}$ is $A$-compact [resp. $\theta A$-compact].

REMARK. The reader may wish to compare Theorem 4.1 with the known 
results that a set $A \subset X$ is compact [resp. quasi- $H$-closed relative to $X$ ] iff ${ }^{*} A$ is $A$-compact [9] [resp. $\theta A$-compact [6]].

Recall that a map $f: X \rightarrow Y$ is strongly $\theta$-continuous at $p \in X$ if for every open neighborhood $N$ of $f(p)$ there exists some open neighborhood $G$ of $p$ such that $f\left[\mathrm{cl}_{X} G\right] \subset N$. Since in the $Q$-topology $\mu(p)$ is open and $\operatorname{cl}^{*} X(\mu(p))$ $=\mu_{\theta}(p)$ for each $p \in X$, then the next result follows easily and compares nicely with the results of Fuller [4] and Wyler [13].

THEOREM 4.2. Let $\mathrm{ns}\left({ }^{*} X\right)\left[\right.$ resp. $\left.\mathrm{ns}_{\theta}\left({ }^{*} X\right)\right]$ carry the topology induced by the $Q$-topology on ${ }^{*} X$. Then St:ns $\left({ }^{*} X\right) \rightarrow X\left[\right.$ resp. $\left.\mathrm{St}_{\theta}: \mathrm{ns}_{\theta}\left({ }^{*} X\right) \rightarrow X\right]$ is a continuous [resp. strongly $\theta$-continuous] map iff $X$ is Hausdorff [resp. Urysohn].

\section{REFERENCES}

1. R. W. Button, Monads for regular and normal spaces (to appear).

2. R. Dickman, $\alpha$-perfect mappings and almost-convergence (preprint).

3. R. Dickman and J. Porter, $\theta$-perfect and $\theta$-absolutely closed functions (preprint).

4. R. V. Fuller, A characterization of local compactness, Proc. Amer. Math. Soc. 37 (1973), 615-616. MR 47 \#525.

5. R. A. Herrmann, Nonstandard topological extensions, Bull. Austral. Math. Soc. 13 (1975), 269-290.

6. - The $\theta$ and $\alpha$-monads in general topology (to appear).

7. W. A. J. Luxemburg, A general theory of monads, Applications of Model Theory to Algebra, Analysis and Probability (Internat. Sympos., Pasadena, Calif., 1967), Holt, Rinehart and Winston, New York, 1969, pp. 18-86. MR 39 \#6244.

8. M. Machover and J. Hirschfeld, Lectures on non-standard analysis, Lecture Notes in Math., vol. 94, Springer-Verlag, Berlin and New York, 1969. MR 40 \#2531.

9. A. Robinson, Non-standard analysis, North-Holland, Amsterdam, 1966.MR 34 \#5680.

10. M. K. Singal and S. P. Arya, On almost-regular spaces, Glasnik Mat. Ser. III 4 (24) (1969), 89-99. MR 39 \#804.

11. - Almost normal and almost completely regular spaces, Glasnik Mat. Ser. III 5 (25) (1970), 141-152. MR 43 \#1111.

12. G. Whyburn, Directed families of sets and closedness of functions, Proc. Nat. Acad. Sci. U.S.A. 54 (1965), 688-692. MR 32 \#435.

13. O. Wyler, A characterization of regularity in topology, Proc. Amer. Math. Soc. 29 (1971), 588-590. MR 43 \#6865.

Department of Mathematics, U.S. Naval Academy, Annapolis, Maryland 21402 